\title{
Narrow Band Imaging
}

National Cancer Institute

\section{Source}

National Cancer Institute. Narrow Band Imaging. NCI Thesaurus. Code C116496.

A high-resolution endoscopic technique that uses two specific wavelengths of blue and green light, chosen for their differing ability to peneatrate tissue. These wavelengths are strongly absorbed by hemoglobin and separately visualize both superficial and deeper vasculature. 